Sādhanā Vol. 37, Part 3, June 2012, pp. 341-349. (c) Indian Academy of Sciences

\title{
Plastic collapse load of corroded steel plates
}

\author{
RAHBAR RANJI AHMAD \\ Department of Ocean Engineering, AmirKabir University of Technology, \\ 15914 Tehran, Iran \\ e-mail: rahbar@aut.ac.ir
}

MS received 8 December 2010; revised 2 January 2012; accepted 6 February 2012

\begin{abstract}
Corrosion is one of the detrimental phenomena which reduces strength of structures. It is common practice to assume a uniform thickness reduction for general corrosion. Since the actual corroded plate has rough surfaces, to estimate the remaining strength of corroded structures, typically a much higher level of accuracy is required. The main aim of present work is to study plastic collapse load of corroded steel plates with irregular surfaces under tension. Non-linear finite element method by using computer code ANSYS was employed to determine plastic collapse load. By comparing the results with uniform thickness assumption, a reduction factor was proposed. It is found that by uniform thickness assumption, plastic collapse load of corroded plates are overestimated.
\end{abstract}

Keywords. Corroded steel plate; plastic collapse; FEM; rough surface.

\section{Introduction}

The cost of maintenance, replacement and the out of service time is very high for corroded structures. It is thus advantageous to develop methods for quick and accurate evaluation of strength for aged structure in service. Corrosion has been mainly responsible for the collapse of some aged ships in the past decades. In view of this, the current standard should be revised. Different types of corrosion can take place; dry or wet corrosion, local or general corrosion. General corrosion is an attack on the metal, when an electrochemical reaction occurs over the entire surface and the thickness is reduced. Thickness reduction amounts to $0.1 \mathrm{~mm}$ per year or higher in marine structures.

Some research works were done on mechanical behaviour of corroded steel plates. For example, Bruneau \& Zahrai (1997) and Zuraski \& Johnson (1990) have studied fatigue strength of corroded steel specimens taken from aged bridges. Chen et al (2005) have studied mechanical properties of low alloy steels in chloride solution. Some research works were also done on structural integrity of aged structures. For example Nakai et al (2006) have studied effect of pitting corrosion on strength of plate elements. Mateus \& Witz (1997) have studied post buckling strength of general corroded plate with one-sided rough surface.

Plastic collapse is one of failure modes which should be examined for strength assessment of aged structures. A common practice to assume a uniform thickness reduction for general 
corroded plate and plastic collapse load is simply calculated by multiplying ultimate stress by residual thickness. It is the main aim of the present work to study plastic collapse load of corroded plates taking into account single and two-sided rough surfaces. For this purpose proposed spectrum for geometry of corroded surface by Rahbar Ranji (2001) based on average and standard deviation of thickness reduction is used to simulate corroded plate with one or both-sided corroded surfaces. Plastic collapse analyses are carried out by using non-linear finite element method (FEM). By comparing the results with strength of corroded plate with flush surface, a reduction factor is proposed.

\section{Geometry of corroded surface}

General corrosion develops in large area and yields rough surface with variable thickness. Rahbar Ranji (2001) has proposed a spectrum for geometry of corroded surface based on average and standard deviation of thickness reduction in the following form

$$
s(k)=\left\{\begin{array}{cc}
\frac{11.88 \alpha \beta \sigma}{k^{2}} \operatorname{Exp}\left[-\frac{2}{3}\left(\frac{\beta}{2.97 \sigma|k|}\right)^{3}\right] \quad \Delta t_{a v r} \leq 2.97 \sigma \\
\frac{\alpha \beta\left(\Delta t_{\text {avr }}+2.97 \sigma\right)^{2}}{\Delta t_{a v r} k^{2}} \operatorname{Exp}\left[-\frac{2}{3}\left(\frac{\beta}{\Delta t_{\text {avr }}|k|}\right)^{3}\right] \Delta t_{\text {avr }}>2.97 \sigma
\end{array}\right.
$$

where $k$ is wave number, $\Delta t_{\text {avr }}$ and $\sigma$ are average and standard deviation of thickness reduction, and $\alpha$ and $\beta$ are two constants which are dependent on corrosion condition and lie in the following ranges

$$
\begin{aligned}
& \alpha=0.01 \sim 0.15 \\
& \beta=0.02 \sim 0.15 .
\end{aligned}
$$

These two constants characterize corrosion conditions and are defined in such a way that statistical characteristic of simulated corroded surface be the same as target surface.

Equation 1 is based on type I asymptotic distribution rule for calculation of extreme values of thickness diminution. Maximum thickness diminution is assumed as extreme largest corrosion depth with cumulative probability of $95 \%$ and minimum thickness diminution is assumed as smallest corrosion depth with cumulative probability of 5\%. Therefore, according to type I asymptotic distribution rule, these values are calculated as follows:

$$
\left\{\begin{array}{l}
\Delta t_{\text {Max }}=\Delta t_{a v r}+2.97 \sigma \\
\Delta t_{\min }=\Delta t_{a v r}-2.97 \sigma
\end{array}\right.
$$

Minimum corrosion depth is defined as uniform corrosion diminution of thickness (figure 1). To express roughness of a different surface parameters are introduced, such as roughness average, root mean square roughness and minimum or maximum value. Maximum valley depth or maximum crest is defined as depth of deepest point from average surface $\left(\Delta t_{\text {Max }}-\Delta t_{\text {avr }}\right)$. Maximum peak height is defined as height of peak point from average surface $\left(\Delta t_{\text {avr }}-\Delta t_{\text {min }}\right)$. The distance between maximum and minimum points of thickness reduction, or crest-to-peak distance is defined as maximum height of surface $\left(\Delta t_{\text {Max }}-\Delta t_{\min }\right)$.

In order to express roughness of surface, a coefficient is defined as ratio of maximum height of surface to average thickness as follows

$$
\text { Roughness coefficient }=\frac{\Delta t_{\text {Max }}-\Delta t_{\min }}{t_{0}-\Delta t_{\min }},
$$

where $t_{0}$ is initial thickness of plate. 


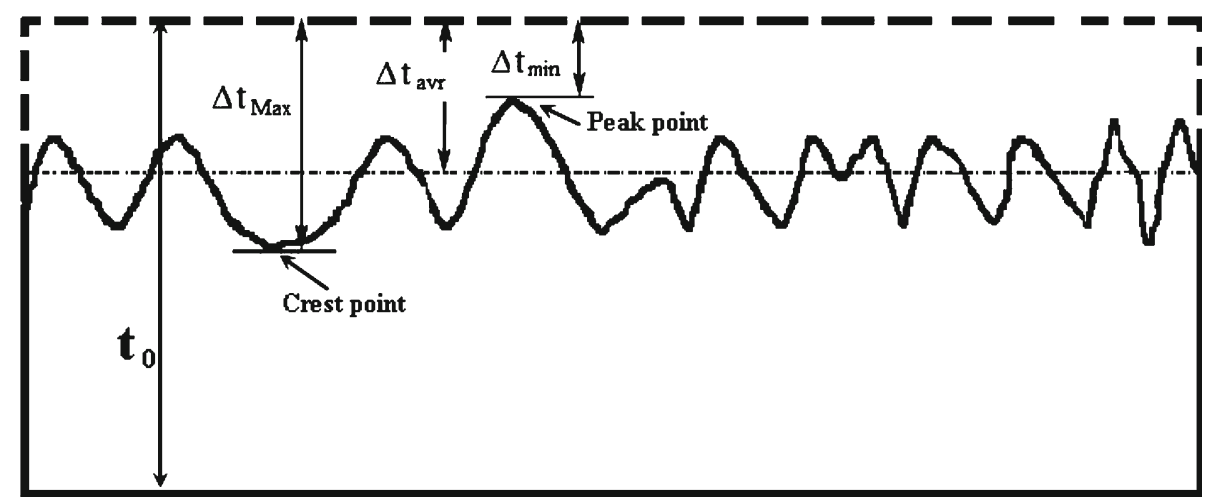

Figure 1. Definition of peak and crest point, $\Delta t_{\mathrm{Max}}, \Delta t_{\mathrm{avr}}$ and $\Delta t_{\min }$ for corroded surface.

Average thickness diminution and standard deviation of thickness are two corrosion parameters which are given for any environment by researchers. Guo et al (2008) have given mean and standard deviation of corrosion wastage in deck plate of single hull tankers as a function of ships age based on measured data. Having mean and standard deviation of thickness diminution, spectrum of corroded surface would be known. Since corrosion is a random phenomenon, stochastic characteristics in all directions should be the same. In another words, energy spectrum of corroded surface is homogeneous in all directions. Therefore, an isotropic spectrum in two directions is expressed by Eq. 1 where equivalent wave number in $x_{1}$ and $x_{2}$ direction is defined as follows

$$
k_{e q}=\sqrt{k_{1}^{2}+k_{2}^{2}} \text {. }
$$

Having 3D spectrum of corroded surface, geometry of corroded surface could be generated from following equation

$$
\begin{aligned}
z\left(x_{1}, x_{2}\right)= & \sqrt{2} \sum_{i=1}^{N_{1}} \sum_{j=1}^{N_{2}} \sqrt{2 S\left(k_{1 i}, k_{2 j}\right) \Delta k_{1} \Delta k_{2}}\left[\operatorname{Cos}\left(k_{1 i} x_{1}+k_{2 j} x_{2}+\varphi_{1 i j}\right)\right. \\
& \left.+\operatorname{Cos}\left(k_{1 i} x_{1}-k_{2 j} x_{2}+\varphi_{2 i j}\right)\right],
\end{aligned}
$$

where $N_{1}$ and $N_{2}$ are number of discretization of spectrum in $x_{1}$ and $x_{2}$ directions, respectively, $\phi_{1 i j}$ and $\phi_{2 i j}$ are random phase angles uniformly distributed between 0 and $2 \pi, \Delta k_{1}$ and $\Delta k_{2}$ are wave number increments in $x_{1}$ and $x_{2}$ directions, respectively and $k_{1 i}=i \Delta k_{1}$ and $k_{2 j}=j \Delta k_{2}$.

\subsection{One-sided corroded plate}

For one-sided corroded plate all thickness reductions occur at one-side of plate. After generating a corroded surface by using Eq. 5, this surface and a flat surface on opposite side are placed in such a way that average and standard deviation of thickness reduction of simulated corroded plate and initial values to be the same.

\subsection{Both-sided corroded plate}

In order to simulate both-sided corroded plate, two undulated surfaces are generated and positioned in such a way that average and standard deviation of thickness reduction of simulated 
plate and initial value be the same. Since corrosion information are limited to thickness reduction rather than surface undulation, some statistical studies are needed to determine how these thickness reduction parameters are divided between two opposite surfaces. Another parameter which has influence on strength of corroded plate is relative position of crest points on each surface.

In this study, both-sided corroded plate with the same thickness reduction parameters at both sides and different thickness reduction parameters at both side with ratio 0.333 and 0.667 are considered. Relative position of two crest points for both-sided plate with different thickness reduction parameters are considered either in the same plane or off-plane.

\section{Plastic collapse analysis}

Elasto-plastic behaviour of material is characterized by an initial elastic response and a plastic deformation (yielding) imposed after a certain level of stress. The stress in which yielding starts is called yield point, and portion of stress-strain curve above this point is called strain hardening portion. The onset of plastic deformation is governed by yield criterion. For uniaxially loaded tensile specimen, yielding is determined simply by comparing axial stress with yield stress. For general states of stress it is not apparent when yielding occurs. Yield criterion is a singular valued and determines stress level at which yielding begins. Different yield criterions have been suggested but Von-Mises criterion fits the experimental results closely.

Work or strain hardening describes how the initial yield criterion changes with progressive straining. Some hardening rules are suggested as below.

- Perfectly plastic which no strain hardening occurs

- Isotropic hardening which assumes yield surface expands in size based on amount of plastic work done in materials

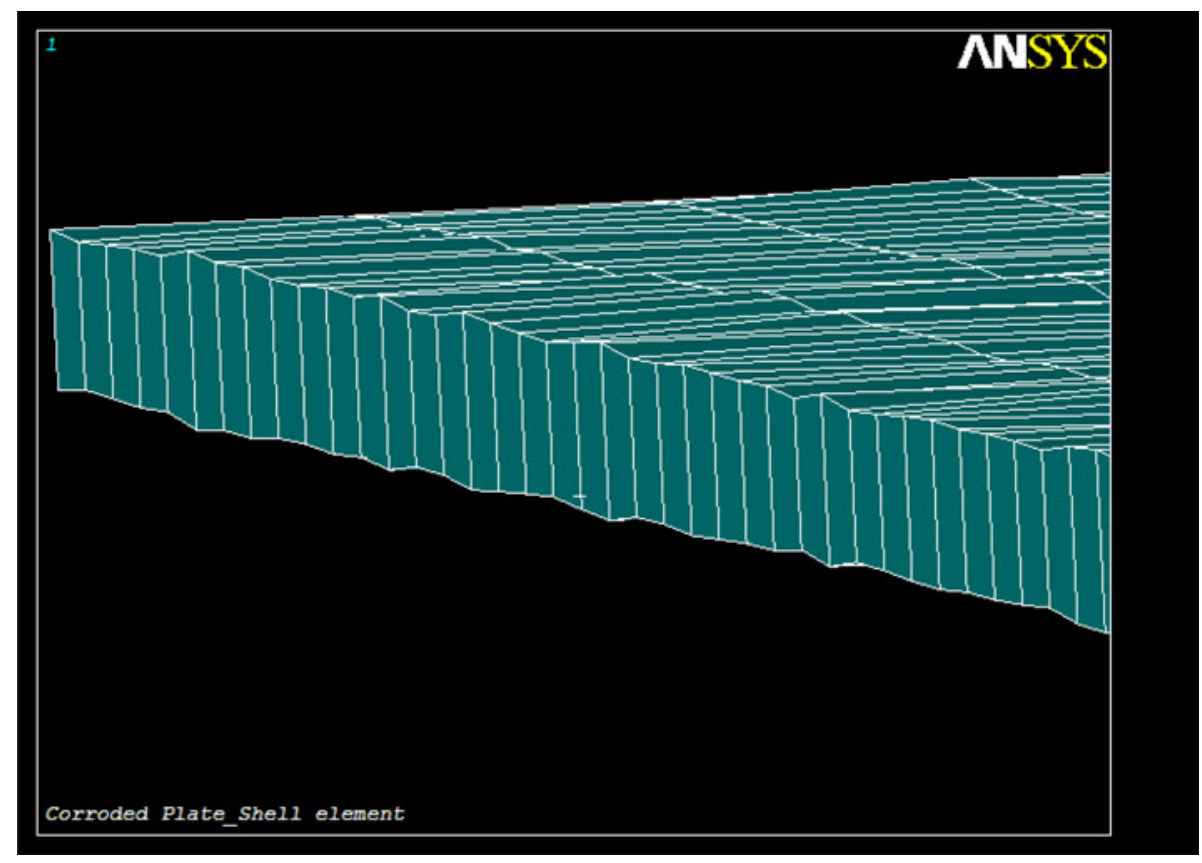

Figure 2. Finite element model of plate with both-sided corroded surfaces (shell elements). 


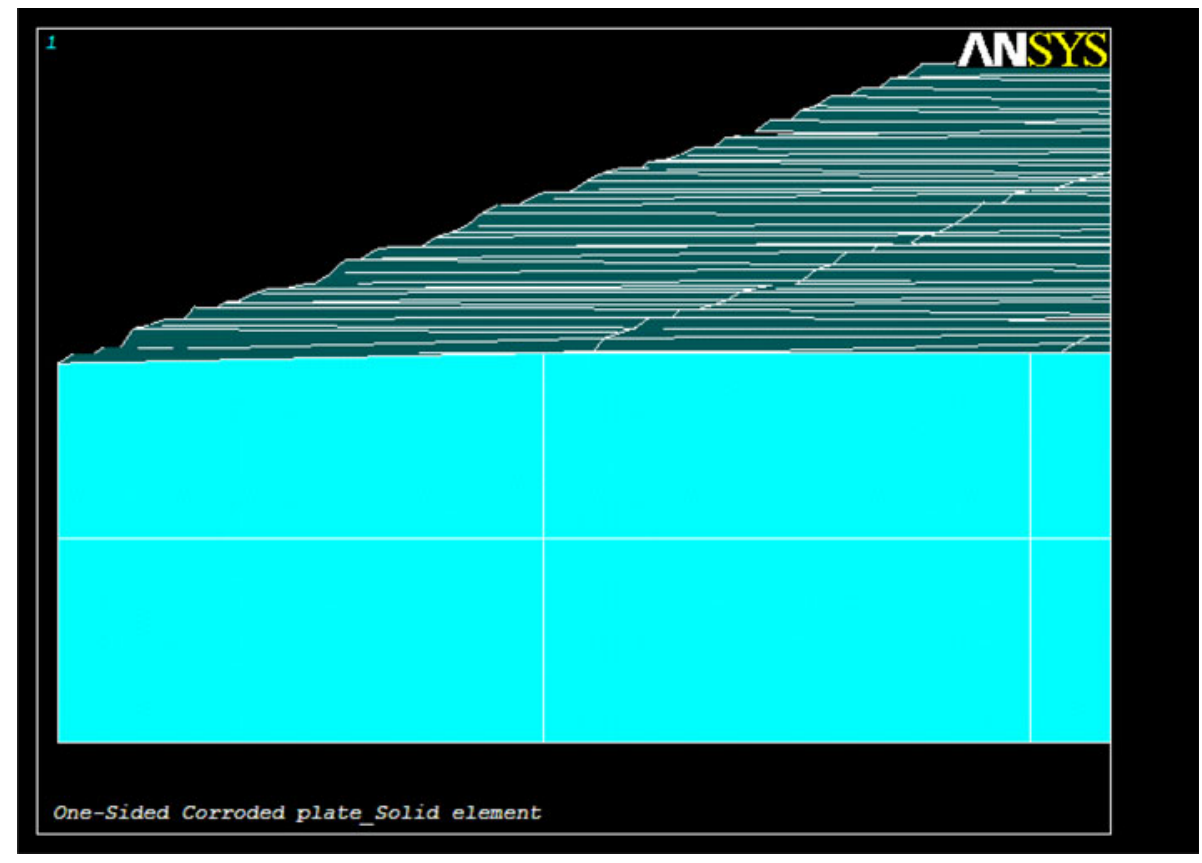

Figure 3. Finite element model of plate with one-sided corroded surfaces (solid elements).

- Kinematic hardening which assumes yield surface remains constant in size but translate in direction of yielding.

Plastic collapse load assessment of corroded plate with irregular surfaces is only on the basis of numerical analysis with FEM. A computer code in Fortran 90 is developed to generate irregular surfaces based on mean and standard deviation of thickness diminution. By using shell elements with variable thickness at each node, both-sided corroded plate with the same thickness reduction parameters at each side is generated. In this case mid-plane of corroded plate is not dislocated (figure 2). Solid elements could be used to model one-sided corroded

Table 1. Reduction factor of plastic collapse load for one-sided corroded plate.

\begin{tabular}{lccc}
\hline Roughness coefficient & Mat.\#1 & Mat.\#2 & Mat.\#3 \\
\hline 0.051 & 1.78 & 1.76 & 1.86 \\
0.063 & 2.10 & 2.14 & 2.33 \\
0.105 & 5.00 & 4.99 & 5.09 \\
0.128 & 5.74 & 5.73 & 5.86 \\
0.147 & 6.63 & 6.50 & 6.65 \\
0.166 & 7.20 & 7.21 & 7.31 \\
0.174 & 8.18 & 8.16 & 8.42 \\
0.175 & 8.27 & 8.25 & 8.45 \\
0.232 & 11.20 & 11.29 & 11.33 \\
0.271 & 12.02 & 12.02 & 12.31 \\
\hline
\end{tabular}


Table 2. Reduction factor of plastic collapse load for both-sided corroded plate, the same thickness reduction parameters at both sides.

\begin{tabular}{lc}
\hline Roughness coefficient & Reduction factor \\
\hline 0.048 & 0.90 \\
0.067 & 2.20 \\
0.098 & 2.90 \\
0.108 & 3.20 \\
0.161 & 5.00 \\
0.173 & 7.20 \\
0.178 & 7.80 \\
0.194 & 6.00 \\
0.201 & 6.40 \\
0.260 & 8.50 \\
\hline
\end{tabular}

plate (figure 3) or both-sided corroded plate with different thickness reduction parameters at both side.

\section{Numerical analysis and discussions}

To study plastic collapse load of corroded surface, geometry of one-sided and both-sided corroded plate with initial thickness $12 \mathrm{~mm}$ are generated with average thickness reduction equals to $1 \mathrm{~mm}$ and standard deviation of thickness diminution equals to $0.2,0.3$ and $0.4 \mathrm{~mm}$. Computer code ANSYS (version 5.6) is used which employs nonlinear finite element method with VonMises yield criterion. Uniform uniaxial tension is applied at one edge of plate and displacement is fixed at opposite edge. To be sure of uniform elongation, a rigid region is created at loaded edge. Plastic collapse load is determined and a reduction factor is defined as follows

$$
R=100 \times \frac{P_{0}-P_{r}}{P_{0}},
$$

Table 3. Reduction factor of plastic collapse load for both-sided corroded plate, different thickness reduction parameters at both sides $(0.333,0.667)$.

\begin{tabular}{lc}
\hline Roughness coefficient & Reduction factor \\
\hline 0.047 & 1.50 \\
0.073 & 2.70 \\
0.098 & 3.20 \\
0.120 & 2.70 \\
0.135 & 4.80 \\
0.190 & 7.0 \\
0.190 & 9.30 \\
0.174 & 6.20 \\
0.221 & 8.70 \\
0.260 & 10.10 \\
\hline
\end{tabular}




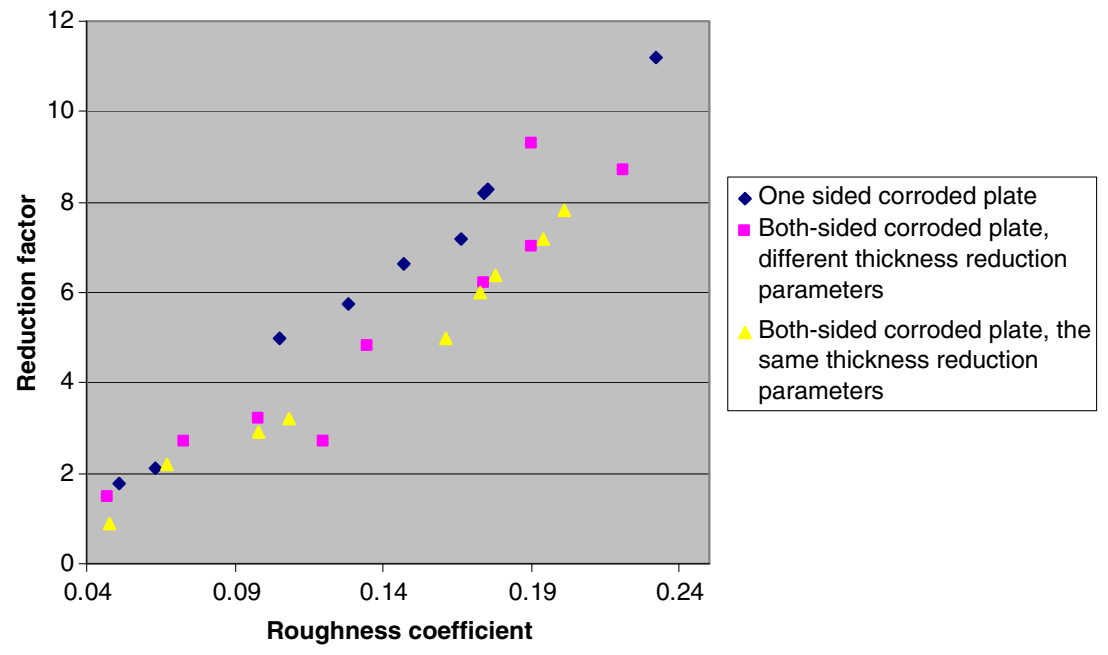

Figure 4. Reduction factor of plastic collapse load in corroded plate.

where $P_{r}$ is plastic collapse load of corroded plate with rough surface and $P_{0}$ is plastic collapse load of corroded plate with flush surface having the same avearge thickness.

\subsection{Two-dimensional models}

In order to increase mesh density and accuracy of calculation, first $2 \mathrm{D}$ models are analysed. Following cases are considered:

(i) One-sided corroded plate

(ii) Both-sided corroded plate with the same thickness reduction parameters at both side

(iii) Both-sided corroded plate with different thickness reduction parameters at both-side (ratio of thickness reduction at both-sides is considered as 0.333 and 0.667 ) and crest points in the same plane

(iv) Both-sided corroded plate with the same thickness reduction parameters and crest points off-plane.

Also, to study the influence of material properties, three different materials for the case of onesided corroded plate are considered.

(i) Elastic-perfectly plastic steel with yield stress $245 \mathrm{MPa}$, (Mat.\#1)

(ii) Elastic-perfectly plastic steel with yield stress $350 \mathrm{MPa}$, (Mat.\#2)

Table 4. Reduction factor of plastic collapse load for both-sided corroded plate, the crest point not in the same plane $(\Delta=5 \mathrm{~mm})$.

\begin{tabular}{lc}
\hline Roughness coefficient & Reduction factor \\
\hline 0.173 & 7.30 \\
0.178 & 7.80 \\
\hline
\end{tabular}


Table 5. Reduction factor of plastic collapse load for both-sided corroded plate, the crest point not in the same plane $(\Delta=10 \mathrm{~mm})$.

\begin{tabular}{lc}
\hline Roughness coefficient & Reduction factor \\
\hline 0.048 & 1.30 \\
0.098 & 3.50 \\
0.161 & 6.70 \\
\hline
\end{tabular}

(iii) Elastic with kinematic hardening with yield stress $245 \mathrm{MPa}$, ultimate strength $367.5 \mathrm{MPa}$ and Tangent Modulus E/10 (Mat.\#3).

In all cases Young's modulus is considered as $206 \mathrm{GPa}$.

In table 1, reduction factor of plastic collapse load for one-sided corroded plate is given. As can be seen, reduction factor increases as roughness coefficient of surface increases and reduction factor for all material types are almost the same regardless of yield stress or type of strain hardening. Therefore in further analysis, yield stress is considered as $245 \mathrm{MPa}$ and elastic-perfectly plastic materials are assumed. In tables 2 and 3, reduction factor for both-sided corroded plate with the same and different thickness reduction parameters at both-sides are given. It can be concluded that for both-sided corroded plate also by increasing roughness coefficient the reduction factor increases. In figure 4 reduction factor for one-sided and both-sided corroded plate as a function of roughness coefficient is shown. As can be seen, reduction factor for the case of one-sided corroded plate is higher than both-sided corroded plate. Even though at some points, reduction factor for both-sided corroded plate with the same thickness reduction parameters are higher than both-sided corroded plate with different thickness reduction at both side, however generally speaking in most cases both-sided corroded plate with different thickness reduction has higher reduction factor. Also it can be concluded that a linear relationship between roughness coefficient and reduction factor of plastic collapse load.

In tables 4 and 5, reduction factor for both-sided corroded plate when deepest point are offplane $(5 \mathrm{~mm}$ and $10 \mathrm{~mm}$ ) are given. Comparing results of tables 4 and 5 with table 2, it can be concluded that relative positions of the crest points at upper and lower surfaces has influence on

Table 6. Reduction factor of plastic collapse load for both-sided corroded plate, 3D model.

\begin{tabular}{lcc}
\hline \multirow{2}{*}{$\begin{array}{l}\text { Roughness } \\
\text { coefficient }\end{array}$} & \multicolumn{2}{c}{ Reduction factor } \\
\cline { 2 - 3 } & Shell element & Solid element \\
\hline 0.109 & 1.34 & 1.18 \\
0.136 & 2.92 & 2.12 \\
0.155 & 2.56 & 2.57 \\
0.162 & 1.62 & 1.10 \\
0.191 & 3.39 & 2.73 \\
0.197 & 2.88 & 3.27 \\
0.200 & 2.90 & 2.86 \\
0.200 & 4.69 & 4.98 \\
0.381 & 3.18 & 3.51 \\
\hline
\end{tabular}


reduction factors, but comparing with one-sided corroded plate, still one-sided corroded plate yields maximum reduction of plastic collapse load.

\subsection{Three-dimensional models}

From 2D analysis it was found that, maximum reduction of plastic collapse load occurs in onesided corroded plate. Therefore, only one-sided corroded plate with yield point $245 \mathrm{MPa}$ and elastic perfectly plastic steel is considered. In table 6, reduction factor for plates with both-sided corroded surface and the same thickness reduction parameters at both-sided by using shell and solid elements are given. For shell element thickness at each node is changed accordingly. As can be seen, almost both cases yield the same results. Comparing results of 2D models with 3D models, it can be concluded that for 3D models, reduction factor is almost half of 2D models. This is because in 2D models plate with infinite width is assumed, deepest point is an infinite line (groove) while in 3D models it is only one point. From table 6, it can be concluded that maximum reduction factor for 3D models is about 5\%. In another words, by uniform thickness assumption for corroded plate, plastic collapse load could be overestimated up to $5 \%$.

\section{Conclusions}

Nonlinear FEM using elastic-plastic material was used for plastic collapse load analysis of corroded plate with one and both-side rough surfaces. A reduction factor is introduced as a ratio of reduction of plastic collapse load of corroded plate with rough surface with respect to plastic collapse load of corroded plate with flush surface. It is found that, one-sided corroded surface has maximum reduction factor. Also, reduction factor almost linearly increases with increase of ratio of maximum height of surface to average thickness. Maximum value of reduction factor for one-sided corroded plate is about $5 \%$. In another words, uniform thickness assumption could lead up to $5 \%$ overestimation of plastic collapse load of corroded plates.

\section{References}

Bruneau M and Zahrai S M 1997 Effect of severe corrosion on cyclic ductility of steel. J. Struct. Eng. 123(11): 1478-1486

Chen Y Y, Tzeng H J, Wei L I and Shih H C 2005 Mechanical properties and corrosion resistance of low-alloy steels in atmospheric conditions containing chlortide. Mat. Sci. Eng. A 398: 47-59

Guo J, Wang G, Ivanov L and Perakis N 2008 Time varying ultimate strength of aging tanker deck plate considering corrosion effect. Mar. Struct. 21: 402-419

Mateus A F and Witz J A 1998 On the post buckling of corroded steel plates used in marine structure. RINA Transactions 140:165-183

Nakai T, Matsushita H and Yamamoto N 2006 Effect of pitting corrosion on the ultimate strength of steel plates subjected to in-plane compression and bending. J. Mar. Sci. Technol. 11: 52-56

Rahbar Ranji Ahmad 2001 Stress analysis of a randomly undulated plate due to corrosion in marine structures. Ph.D. thesis, Yokohama National University

Zuraski P D and Johnson J E 1990 Fatigue strength of deteriorated steel highway bridges. J. Struct. Eng. 116(10): 2671-2690 